\title{
Comparison of three different kits for extraction of high-quality RNA from frozen blood
}

\author{
Jin-Hee Kim ${ }^{1}$, Hyeon-Ok Jin', Jin-Ah Park', Yoon Hwan Chang ${ }^{2}$, Young Jun Hong ${ }^{2}$ and Jin Kyung Lee ${ }^{1,2^{*}}$
}

\begin{abstract}
Extraction of high-quality RNA is a crucial step in gene expression profiling. To achieve optimal RNA extraction from frozen blood, the performance of three RNA extraction kits- TRI reagent, PAXgene blood RNA system (PAXgene) and NucleoSpin RNA blood kit (NucleoSpin)- was evaluated. Fifteen blood specimens collected in tubes containing potassium ethylenediaminetetraacetic acid (EDTA) and stored at $-80^{\circ} \mathrm{C}$ for approximately 5 years were randomly selected. The yield and purity of RNA, RIN (RNA integrity number) values and cycle threshold (Ct) values were assessed. Mean RNA yields with TRI reagent, PAXgene and NucleoSpin were $15.6 \pm 8.7 \mu \mathrm{g} / \mathrm{ml}, 3.1 \pm 1.7 \mu \mathrm{g} / \mathrm{ml}$ and $9.0 \pm 5.5 \mu \mathrm{g} / \mathrm{ml}$, respectively. Mean $A_{260} / 280$ ratios of RNA for the three kits were $1.7 \pm 0.1,2.0 \pm 0.1$, and $2.0 \pm 0.0$, and mean RIN values recorded as $3.2 \pm 0.8,6.0 \pm 1.1$, and $6.4 \pm 0.9$, respectively. The Ct values of housekeeping genes, $18 \mathrm{~S}$ rRNA, $\beta$-actin, RPLPO and HPRT1, were as follows: TRI reagent (19.2 $\pm 1.6,30.6 \pm 1.8,29.9 \pm 1.4$ and $36.3 \pm 1.3)$, PAXgene $16.6 \pm 1.4,26.4 \pm 1.3,28.2 \pm 1.8$ and $33.8 \pm 1.1)$, and NucleoSpin $(16.3 \pm 1.5,27.2 \pm 1.3,27.0 \pm 1.6$ and $32.9 \pm$ 1.6). RNA yield using TRI reagent was 1.7 times higher than that with NucleoSpin and 5 times higher than that with PAXgene. However, the purity and integrity of TRI-extracted RNA was lower than that extracted with PAXgene and NucleoSpin. Moreover, the Ct values of housekeeping genes after extraction with TRI reagent were approximately 1.7-3.8 times higher than those obtained with PAXgene and NucleoSpin. The PAXgene and NucleoSpin kits produced similar results in terms of RNA purity and integrity and subsequent gene amplification. However, RNA yields from NucleoSpin were 2.9-fold higher, compared to PAXgene. Based on these findings, we conclude that NucleoSpin is the most effective kit for extraction of abundant and high-quality RNA from frozen blood.
\end{abstract}

Keywords: Frozen blood; RNA extraction; RT-qPCR

\section{Background}

Human blood specimens are routinely collected by biobanks for medical applications, scientific research, and diagnostic purposes (Elliott and Peakman 2008; Jackson and Banks 2010; Hebels et al. 2013). Blood specimens collected in tubes containing anticoagulants are usually processed to produce aliquots of whole blood, serum, plasma, or buffy coats, which are stored at $-80^{\circ} \mathrm{C}$ for future use. Frozen blood samples stored in biobanks are increasingly considered valuable sources of RNA for profiling gene expression (Barnes et al. 2010). However, a major limitation of studies using RNA isolated from

\footnotetext{
* Correspondence: jklee@kirams.re.kr

'KIRAMS Radiation Blood Specimen Biobank, Korea Institute of Radiological \& Medical Sciences, 215-4 Gongneung-dong, Nowon-gu, Seoul 139-709, Republic of Korea

${ }^{2}$ Department of Laboratory Medicine, Korea Cancer Center Hospital, Korea Institute of Radiological \& Medical Sciences, 215-4 Gongneung-dong, Nowon-gu, Seoul 139-709, Republic of Korea
}

frozen blood samples is RNA degradation during blood collection and storage.

To minimize the degradation of RNA and changes in gene expression patterns, improved alternatives for collecting human blood specimens have been developed (Chai et al. 2005). For instance, PaxGene Blood RNA tubes (PAXgene, PreAanlytix, Hombrechtikon, Switzerland) containing RNA stabilizing reagents are used to collect and store blood samples prior to RNA isolation. Despite the availability of an RNA stabilization system, there may be a need to perform RNA expression profiling experiments with legacy blood samples initially collected using common blood collection tubes (e.g. EDTA tubes) (Chai et al. 2005; Thörn et al. 2005). Recently, Beekman and co-workers proposed an effective way of extracting RNA from frozen blood samples collected using EDTA tubes. Thawed blood is transferred to PAXgene Blood RNA tubes, and RNA 
obtained using the PAXgene RNA extraction kit. This transfer method yields abundant RNA of sufficient quality for gene expression analysis (Beekman et al. 2009).

The most common RNA extraction kits for frozen blood are based on the use of an acid guanidinium thiocyanate-phenol-chloroform extraction reagent. The TRI reagent (Invitrogen, Carlsbad, CA, USA) is based on this technology and rapidly inhibits RNase activity, making it a complete, ready-to-use reagent for total RNA extraction (Kang et al. 2011; Jakovljevic et al. 2010).

Another product, the NucleoSpin RNA Blood kit (NucleoSpin, MACHEREY-NAGEL, Duren, Germany), facilitates extraction of RNA from blood samples collected in EDTA blood collection tubes.

In the current study, three different RNA extraction kits, specifically, TRI reagent, PAXgene, and NucleoSpin, were compared in terms of RNA yield, purity, and integrity, with a view to achieving optimal high-quality RNA extraction from frozen whole blood. RNA extracted with the three kits was additionally assessed for use in realtime PCR assays.

\section{Results and discussion}

The mean yields of RNA isolated from 15 frozen whole blood samples with the three different kits were as follows: TRI reagent, $15.6 \pm 8.7 \mu \mathrm{g} / \mathrm{ml}$ (range, $6.1 \sim 37.6$ ), PAXgene, $3.1 \pm 1.7 \mu \mathrm{g} / \mathrm{ml}$ (range, $1.1 \sim 6.9$ ), and NucleoSpin, $9.0 \pm$ 5.5 (range, $3.6 \sim 23.7) \mu \mathrm{g} / \mathrm{ml}$ (Table 1 and Figure 1A). TRI reagent yielded the highest quantity, generally resulting in extraction of 5.0-fold more RNA than the PAXgene kit and 1.7-fold more RNA than the NucleoSpin kit. However, RNA extracted with TRI reagent exhibited lower purity than that obtained using either PAXgene or NucleoSpin (Table 1 and Figure 1B). RNA absorbs UV light maximally at $260 \mathrm{~nm}$ and protein at $280 \mathrm{~nm}$, and $\mathrm{A}_{260} / \mathrm{A}_{280}$ ratios are thus used to indicate RNA sample purity. The mean RNA purity $\left(\mathrm{A}_{260} / \mathrm{A}_{280}\right)$ was evaluated as $1.7 \pm 0.1$ with TRI reagent, $2.0 \pm 0.1$ with PAXgene, and $2.0 \pm 0.0$ with NucleoSpin. Generally, samples with ratios in the range of $1.8 \sim 2.0$ indicate higher levels of RNA purity. The $A_{260} / A_{280}$ ratios for PAXgene and NucleoSpin extractions were within the desired range, while the ratio for TRI reagent was somewhat lower, indicative of protein or DNA contamination with this kit. As a secondary measurement of RNA purity, $\mathrm{A}_{260} / \mathrm{A}_{230}$ ratios were additionally evaluated for extracts with TRI reagent $(1.4 \pm 0.4)$, PAXgene $(1.1 \pm 0.3)$, and NucleoSpin $(1.8 \pm 0.2)$ (Table 1 and Figure $1 C$ ). The mean $A_{260} / A_{230}$ ratios for RNA obtained with PAXgene and TRI reagent were lower than that with Nucleospin. Phenol, carbohydrate and salt contamination are reported to induce a decrease in the $A_{260} / A_{230}$ ratio. In this regard, the lower $A_{260} / A_{230}$ values may be attributed to the high salt content of PAXgene elution buffer and phenol in TRI reagent.

RNA integrity was assessed using an Agilent 2100 BioAnalyzer, which provides RIN scores for RNA quality control. The mean RIN values were $3.2 \pm 0.8$ for TRI reagent, $6.0 \pm 1.1$ for PAXgene, and $6.4 \pm 0.9$ for NucleoSpin (Table 1 and Figure 1D), signifying that the PAXgene and NucleoSpin kits yield RNA with better integrity than the TRI reagent.

To further validate the quality of RNA obtained using the three different extraction kits, real-time PCR analysis was performed. The expression patterns of four housekeeping genes, 18S rRNA, $\beta$-actin, RPLPO and HPRT1, were analyzed and presented as cycle threshold $(\mathrm{Ct})$ values. As shown in Figure 2, the $\mathrm{Ct}$ values obtained from TRI reagent, PAXgene and NucleoSpin extractions were $19.2 \pm 1.6,16.6 \pm 1.4$ and $16.3 \pm 1.5$ for $18 S r R N A, 30.6 \pm$ $1.8,26.4 \pm 1.3$ and $27.2 \pm 1.3$ for $\beta$-actin, $29.9 \pm 1.4,28.2 \pm$ 1.8 and $27.0 \pm 1.6$ for $R P L P O$, and $36.3 \pm 1.3,33.8 \pm 1.1$ and $32.9 \pm 1.6$ for $H P R T 1$, respectively. The Ct values for RNA extracted using TRI reagent were $\sim 1.7$ to 3.8 times higher than those evaluated with the PAXgene and NucleoSpin kits, clearly indicating that RNA extracted with TRI reagent is of lower quality.

Among the three kits, TRI reagent produced the highest RNA yield. However, the purity of TRI-extracted RNA $\left(\mathrm{A}_{260} / 280\right.$ ratio of 1.7$)$ and integrity (RIN 3.2) were lower, compared to RNA extracted using the PAXgene $\left(\mathrm{A}_{260} /_{280}\right.$ ratio 2.0, RIN 6.0) and NucleoSpin $\left(\mathrm{A}_{260} / 280\right.$ ratio 2.0, RIN 6.4) systems. Earlier, Fleige and co-workers reported that RNA with low RIN values shows reduced PCR performance due to the presence of more highly fragmented molecules, leading to inaccurate gene expression evaluation (Fleige and Pfaffl 2006, Fleige et al. 2006). Gene expression levels of $c$-fos, $I L-1 \beta, I L-8$, and GAPDH were decreased in blood samples with RIN values below 5 (Pazzagli et al. 2013). RIN values of the RNA extracted with PAXgene and NucleoSpin were

Table 1 Yield, purity, and integrity of total RNA isolated from frozen blood using three different extraction kits: TRI reagent, PAXgene, and NucleoSpin $(n=15)$

\begin{tabular}{lccc}
\hline & RNA yield $(\boldsymbol{\mu g} / \mathbf{m l})$ & RNA purity (A260/A280) (A260/A230) & RNA integrity (RIN) \\
\hline TRI reagent & $15.6 \pm 8.7(6.1-37.6)$ & $1.7 \pm 0.1(1.6-1.7) 1.4 \pm 0.4(0.7-2.1)$ & $3.2 \pm 0.8(1.6-5.0)$ \\
PAXgene & $3.1 \pm 1.7(1.1-6.9)$ & $2.0 \pm 0.1(1.9-2.1) 1.1 \pm 0.3(0.6-1.5)$ & $6.0 \pm 1.1(4.0-7.7)$ \\
NucleoSpin & $9.0 \pm 5.5(3.6-23.7)$ & $2.0 \pm 0.0(2.0-2.1) 1.8 \pm 0.2(1.5-2.1)$ & $6.4 \pm 0.9(4.6-7.6)$ \\
\hline
\end{tabular}

Data are presented as means \pm standard deviation. Numbers in parentheses represent the data range. 

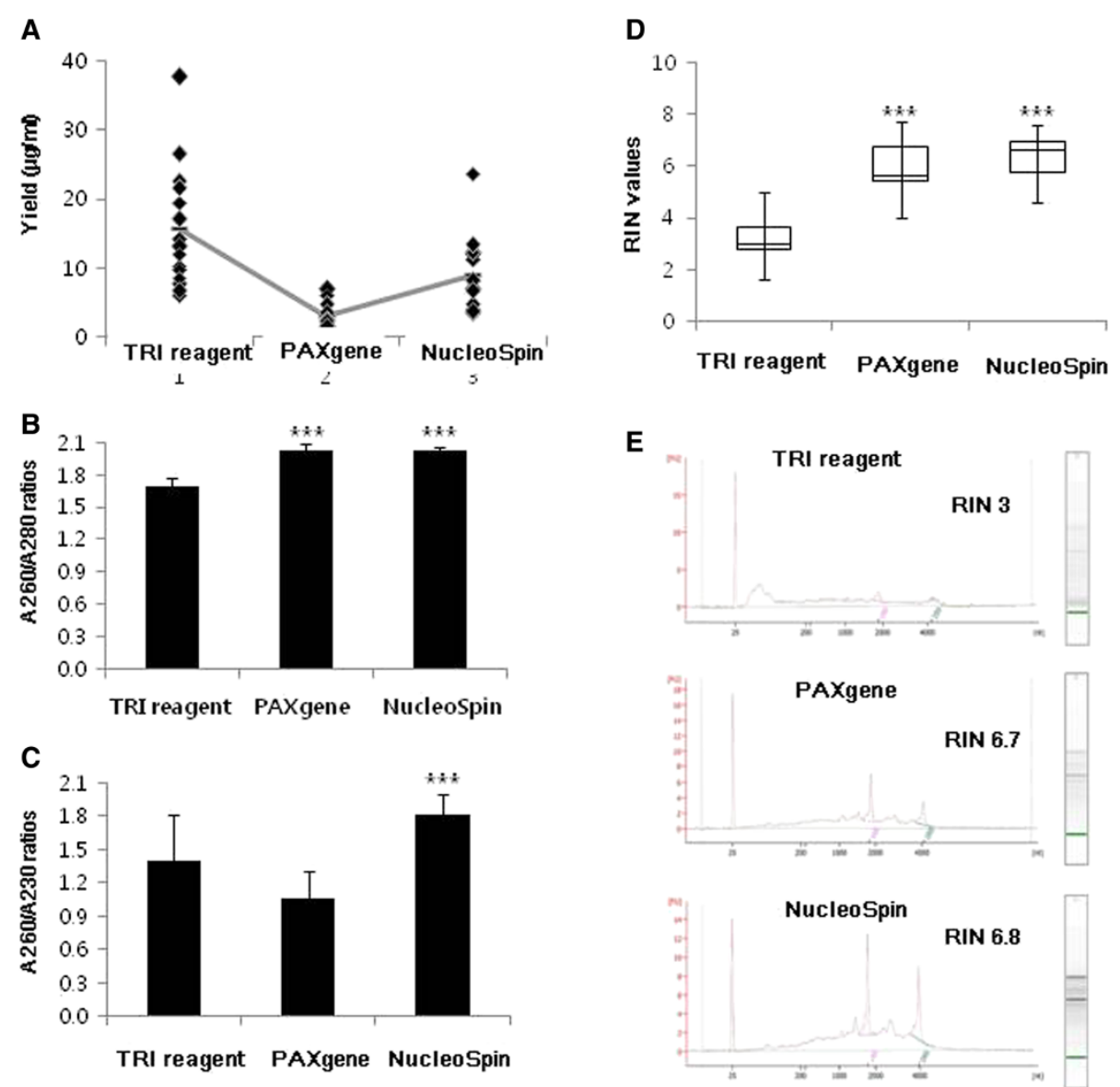

$\mathbf{E}$
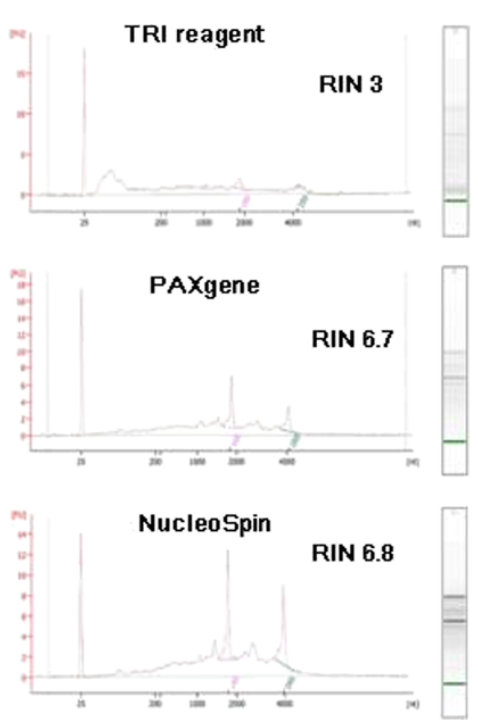

Figure 1 Yield, purity, and integrity of RNA isolated from frozen blood using three different extraction kits. (A) The graph shows individual yields $(n=15)$. $(\mathbf{B}, \mathbf{C})$ The graphs show mean $A_{260} / A_{280}$ and $A_{260} / A_{230}$ ratios. Bars indicate standard deviations. (D) The graph depicts a box-and-whisker plot of variations in RIN values. The upper and lower edges of the boxes represent the $25 \%$ and $75 \%$ lines, and the central line shows median values. (E) RNA was run on a eukaryote total RNA Nano chip. $18 \mathrm{~S}$ and $28 \mathrm{~S}$ peaks of sample \#2 are shown. ${ }^{* * *} p<0.001$ versus TRI reagent (b). ${ }^{* *} p<0.001$ versus TRI reagent or PAXgene $(\mathbf{C})$.

higher than the cut-off value of 5 , suggesting that RNA extracted using these kits is of similar quality and performs well in standard gene expression analysis (Beekman et al. 2009; Pazzagli et al. 2013). In the current study, data obtained with real-time PCR revealed lower Ct values of the housekeeping genes, $18 S$ rRNA, $\beta$-actin, RPLPO and HPRT1, measured from PAXgene and NucleoSpin extractions, compared to those measured from TRI reagentextracted RNA (Figure 2). RNA extracted with TRI reagent can contain trace genomic DNA contamination, which presents significant problems, especially for PCR-based applications (Jiang et al. 2013). In our experiments, RNA isolated using TRI reagent was not treated with DNase I. Thus, we propose that TRI reagent-extracted RNA is contaminated with genomic DNA, thereby showing high RNA yield that is overestimated, low in purity and poorly amplified.

The increased availability of blood collection systems with RNA-stabilizing additives and accompanying RNA extraction kits has significantly improved the quality of RNA isolated from blood (Rainen et al. 2002; Thach et al. 2003). However, there remains a need to assess RNA quality and profiling experiments in blood specimens collected with common blood collection systems (EDTA tubes). Beekman and co-workers proposed a transfer method of thawed EDTA blood into the PAXgene system, which is efficient in terms of yield and quality of RNA, compared to the standard protocol (QIAamp RNA blood kit, Qiagen, Valencia, CA, USA) (Beekman et al. 2009). However, this method could not be expected to reverse gene expression changes and variability or RNA quality loss that may have occurred after blood samples originally collected without RNA-stabilizing additives (Beekman et al. 2009).

The PAXgene and NucleoSpin kits produced similar results in terms of RNA purity and integrity and subsequent gene amplification. Our results indicate that RNA extracted from frozen blood samples using the PAXgene 

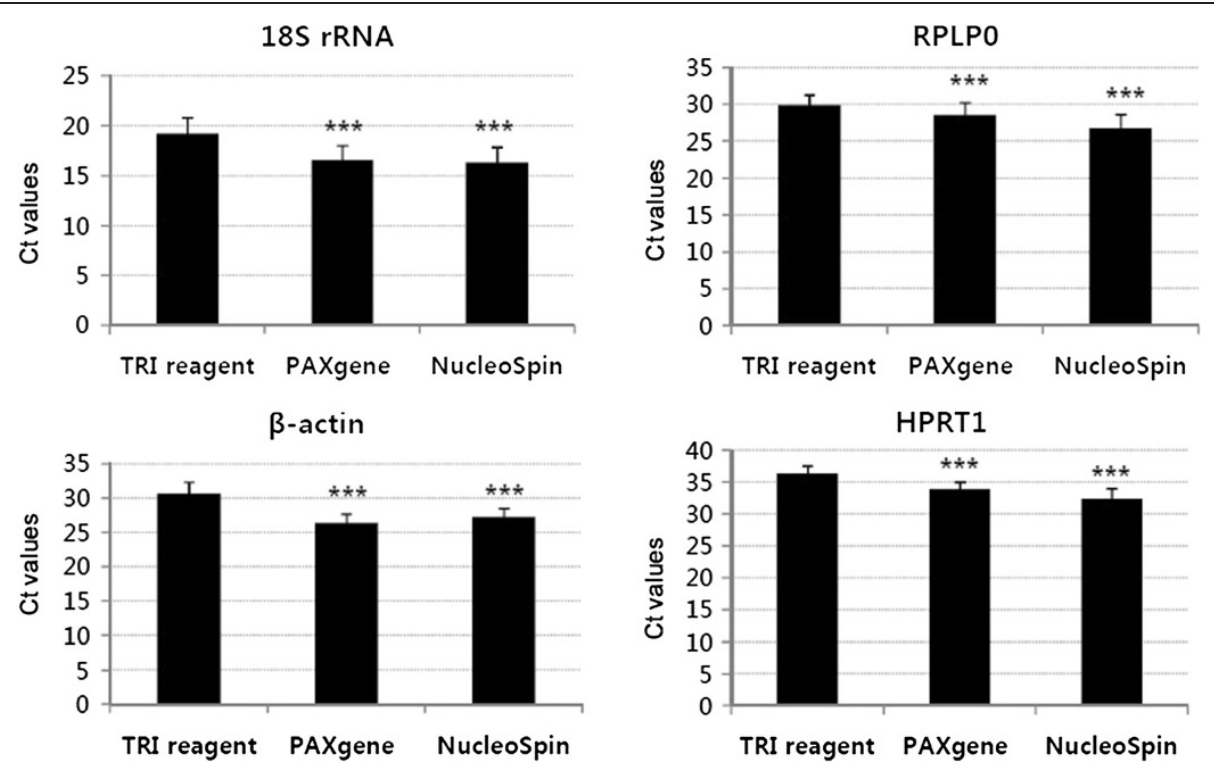

Figure 2 Graph of cycle thresholds (Ct) of three housekeeping genes assessed for RNA samples extracted using three different kits (TRI reagent, PAXgene, and NucleoSpin) and amplified using real time RT-PCR $(\mathbf{n}=\mathbf{1 5})$. Bars indicate standard deviations. ${ }^{* * *} p<0.001$ versus TRI reagent.

and NucleoSpin kits is of high quality, which should facilitate gene expression analyses, such as the real-time PCR assay. However, the RNA yield from NucleoSpin was higher than that from PAXgene. Based on the collective findings, we conclude that extraction using the NucleoSpin kit is the most effective way to produce abundant and high-quality RNA from frozen blood.

\section{Materials and methods}

\section{Samples and RNA extraction procedure}

Blood specimens were collected with informed consent from cancer patients undergoing radiation treatment in Korea Cancer Center Hospital. Blood was collected into potassium EDTA tubes (Greiner Bio-One, Frickenhausen, Germany), transferred to Axygen microtubes (Corning, Tewsbury, USA), and stored at $-80^{\circ} \mathrm{C}$. After approval of the Institutional Review Board, 15 frozen blood specimens stored for approximately 5 years were randomly selected.

Frozen blood specimens were thawed on ice and RNA extracted using three different kits (TRI reagent, PAXgene and NucleoSpin) according to the respective manufacturer protocols.

For TRI reagent extraction, $200 \mu \mathrm{l}$ of each blood sample was incubated with $800 \mu \mathrm{l}$ TRI reagent for $5 \mathrm{~min}$ at room temperature, followed by supplementation with $200 \mu \mathrm{l}$ chloroform. After vigorous mixing and centrifugation at $12,000 \mathrm{~g}$ for $15 \mathrm{~min}$ at $4^{\circ} \mathrm{C}$, the upper layer was transferred to a new tube. An aliquot of $500 \mu \mathrm{l}$ isopropanol was added and the resulting mixture incubated for $10 \mathrm{~min}$ at room temperature, followed by centrifugation at $12,000 \mathrm{~g}$ for $15 \mathrm{~min}$ at $4^{\circ} \mathrm{C}$ to pellet
RNA. The pellet was washed with $1 \mathrm{ml} 75 \%$ ethanol and air-dried for $5 \mathrm{~min}$. RNA was dissolved in $40 \mu \mathrm{l}$ RNase-free water.

For PAXgene extraction, $300 \mu \mathrm{l}$ of each blood sample was dispensed into $830 \mu \mathrm{l}$ PAXgene reagent at a blood: reagent ratio of 2.5:6.9, which was the same as in the PAXgene Blood RNA Tubes (Beekman et al. 2009; Carrol et al. 2007; Krawiec et al. 2009). The mixture was incubated for $16 \mathrm{~h}$ at room temperature. After washing with $500 \mu \mathrm{l}$ RNase-free water, the pellet was dissolved in $350 \mu \mathrm{l}$ resuspension buffer and incubated with $300 \mu \mathrm{l}$ binding buffer and $40 \mu \mathrm{l}$ proteinase $\mathrm{K}$ for $10 \mathrm{~min}$ at $55^{\circ} \mathrm{C}$ in a shaker-incubator. The lysate was transferred into a PAXgene shredder spin column and centrifuged (at 18,000 g for $3 \mathrm{~min}$ ). The flow-through fraction was mixed with $350 \mu \mathrm{l}$ ethanol and transferred to a PAXgene RNA spin column. After washing the column with washing buffer 1 , samples were incubated with $10 \mu \mathrm{l}$ of DNase I for 15 min. PAXgene RNA spin columns were washed with washing buffer and RNA eluted with $40 \mu$ l elution buffer.

For NucleoSpin extraction, $200 \mu \mathrm{l}$ of each blood sample was transferred to a collection tube to which $200 \mu$ lysis buffer DL and $5 \mu$ l Proteinase K were added. The mixture was incubated for $15 \mathrm{~min}$ with vigorous shaking. Lysates were mixed with $200 \mu \mathrm{l}$ of $70 \%$ ethanol, transferred to a NucleoSpin RNA Blood column, and washed with $350 \mu \mathrm{l}$ MDB. Subsequently, $95 \mu \mathrm{l}$ of rDNase was added to the column and incubated at room temperature for $15 \mathrm{~min}$. The column was washed with Buffers RB2 and RB3, and RNA eluted using $40 \mu \mathrm{l}$ RNase-free water. 
RNA assessment (yield, purity and integrity)

The RNA yield was estimated by measuring absorbance at $260 \mathrm{~nm}$ in a NanoDrop 2000 spectrophotometer (Thermo Fisher Scientific, Wilmington, DE, USA). RNA purity was calculated from the ratio of absorbance at $260 \mathrm{~nm}$ and $280 \mathrm{~nm}$, and integrity assessed using the Eukaryote Total RNA Nano assay on the Agilent 2100 Bioanalyzer (Agilent Technologies, Santa Clara, CA, USA). The RNA integrity number (RIN) was calculated using the Agilent 2100 Bioanalyzer and accompanying software.

\section{RT-PCR analysis}

cDNA was reverse-transcribed from total RNA using SuperScript II RNase transcriptase (Invitrogen, Carlsbad, CA, USA) following the manufacturer's guidelines. In total, 200 ng of total RNA was used as template. The reaction mixtures were incubated in a 2720 Thermal Cycler (Applied Biosystems, Foster City, CA, USA). TaqMan Gene Expression Master Mix was used to perform real-time PCR (Applied Biosystems, Foster City, CA, USA). $18 S$ rRNA (Hs99999901_s1), $\beta$-actin (ACTB, Hs99999903_m1), RPLPO (Hs99999902_m1) and HPRT1 (Hs99999909_m1) genes were amplified and quantified. Each reaction mixture $(20 \mu \mathrm{l})$ was transferred to a 96well plate, which was loaded onto the 7500 Real Time PCR System (Applied Biosystems, Foster City, CA, USA). Plates were heated to $50^{\circ} \mathrm{C}$ for 2 min and $95^{\circ} \mathrm{C}$ for $10 \mathrm{~min}$, and subjected to cycles of $95^{\circ} \mathrm{C}$ for $15 \mathrm{sec}$ and $60^{\circ} \mathrm{C}$ for $1 \mathrm{~min}$. Data were expressed as cycle threshold $(\mathrm{Ct})$ values.

\section{Statistical analysis}

Experimental data are expressed as mean values and standard deviation. The performance of the three RNA extraction kits was compared with the Student's $t$-test. Asterisks $(* * * * 0.001)$ represent statistically significant differences.

\section{Competing interests}

The authors declare that they have no competing interests.

\section{Authors' contributions}

JK and $Y J$ initiated and designed the work. JH and JA carried out the experiments. $\mathrm{HO}$ and $\mathrm{YH}$ assisted in manuscript preparation. All authors read and approved the final version of the manuscript.

\section{References}

Barnes MG, Grom AA, Griffin TA, Colbert RA, Thompson SD (2010) Gene expression profiles from peripheral blood mononuclear cells Are sensitive to short processing delays. Biopresery Biobank 8:153-162

Beekman JM, Reischl J, Henderson D, Bauer D, Ternes R, Peña C, Lathia C, Heubach JF (2009) Recovery of microarray-quality RNA from frozen EDTA blood samples. J Pharmacol Toxicol Methods 59:44-49

Carrol ED, Salway F, Pepper SD, Saunders E, Mankhambo LA, Ollier WE, Hart CA, Day P (2007) Successful downstream application of the Paxgene Blood RNA system from small blood samples in paediatric patients for quantitative PCR analysis. BMC Immunol 8:20

Chai V, Vassilakos A, Lee Y, Wright JA, Young AH (2005) Optimization of the PAXgene blood RNA extraction system for gene expression analysis of clinical samples. J Clin Lab Anal 19:182-188

Elliott P, Peakman TC (2008) The UK Biobank sample handling and storage protocol for the collection, processing and archiving of human blood and urine. Int J Epidemiol 37:234-244

Fleige S, Pfaffl MW (2006) RNA integrity and the effect on the real-time qRT-PCR performance. Mol Aspects Med 27:126-139

Fleige S, Walf V, Huch S, Prgomet C, Sehm J, Pfaffl MW (2006) Comparison of relative $m R N A$ quantification models and the impact of RNA integrity in quantitative real-time RT-PCR. Biotechnol Lett 28:1601-1613

Hebels DG, Georgiadis P, Keun HC, Athersuch TJ, Vineis P, Vermeulen R, Portengen L, Bergdahl IA, Hallmans G, Palli D, Bendinelli B, Krogh V, Tumino R, Sacerdote C, Panico S, Kleinjans JC, de Kok TM, Smith MT, Kyrtopoulos SA (2013) Performance in omics analyses of blood samples in long-term storage: opportunities for the exploitation of existing biobanks in environmental health research. Environ Health Perspect 121:480-487

Jackson DH, Banks RE (2010) Banking of clinical samples for proteomic biomarker studies: a consideration of logistical issues with a focus on pre-analytical variation. Proteomics Clin Appl 4:250-270

Jakovljevic KV, Spaxic MR, Malisic EJ, Dobricic JD, Krivokuca AM, Jankovic RN (2010) Comparison of phenol-based and alternative RNA isolation methods for gene expression analyses. J Serb Chem Soc 75:1053-1061

Jiang Z, Uboh CE, Chen J, Soma LR (2013) Isolation of RNA from equine peripheral blood cells: comparison of methods. Springerplus 2:478

Kang JE, Hwang SH, Lee JH, Park do Y, Kim HH (2011) Effects of RBC removal and TRIzol of peripheral blood samples on RNA stability. Clin Chim Acta 412:1883-1885

Krawiec JA, Chen H, Alom-Ruiz S, Jaye M (2009) Modified PAXgene method allows for isolation of high-integrity total RNA from microlitre volumes of mouse whole blood. Lab Anim 43:394-398

Pazzagli M, Malentacchi F, Simi L, Orlando C, Wyrich R, Günther K, Hartmann CC, Verderio P, Pizzamiglio S, Ciniselli CM, Tichopad A, Kubista M, Gelmini S (2013) SPIDIA-RNA: first external quality assessment for the pre-analytical phase of blood samples used for RNA based analyses. Methods 59:20-31

Rainen L, Oelmueller U, Jurgensen S, Wyrich R, Ballas C, Schram J, Herdman C, Bankaitis-Davis D, Nicholls N, Trollinger D, Tryon V (2002) Stabilization of mRNA expression in whole blood samples. Clin Chem 48:1883-1890

Thach DC, Lin B, Walter E, Kruzelock R, Rowley RK, Tibbetts C, Stenger DA (2003) Assessment of two methods for handling blood in collection tubes with RNA stabilizing agent for surveillance of gene expression profiles with high density microarrays. J Immunol Methods 283:269-279

Thörn I, Olsson-Strömberg U, Ohlsen C, Jonsson AM, Klangby U, Simonsson B, Barbany G (2005) The impact of RNA stabilization on minimal residual disease assessment in chronic myeloid leukemia. Haematologica 90:1471-1476

doi:10.1186/2193-1801-3-76

Cite this article as: Kim et al:: Comparison of three different kits for extraction of high-quality RNA from frozen blood. SpringerPlus 2014 3:76.

\author{
Acknowledgements \\ The specimens used for this study were distributed by the Korea Institute of \\ Radiological and Medical Sciences (KIRAMS) Radiation Blood Specimen \\ Biobank (KRBB). The study was approved by the Institutional Review Board \\ (IRB No. K-1304-002-049), and supported by a grant from the Radiation \\ Bio-Resource Research Program of the Korea Institute of Radiological and \\ Medical Sciences (No. 740802), Republic of Korea.
}

Received: 12 November 2013 Accepted: 5 February 2014

Published: 8 February 2014 